\title{
Pre-Descemet corneal dystrophy
}

INSERM

\section{Source}

INSERM. (1999). Orphanet: an online rare disease and orphan drug data base. Pre-

Descemet corneal dystrophy. ORPHA:293462

Pre-Descemet corneal dystrophy (PDCD) is a rare form of stromal corneal dystrophy characterized by focal, fine, gray opacities in the deep stroma immediately anterior to the Descemet membrane, with no effect on vision. 